\title{
Use of Polyguanidine-Derivatives-Based Biocides for Microbial Growth Inhibition and for the Development of a Novel Polyethylene-Based Composite Material Resistant to the Formation of Multispecies Microbial Biofilms
}

\author{
Marina Zhurina1, Andrei Gannesen¹, Sergey Martyanov¹, Anna Kallistova, \\ Victor Gerasin², Nikolai Sivov², Vsevolod Tikhomirov², Ahkar Kaung Myint ${ }^{2}$, \\ and Vladimir Plakunov ${ }^{1}$ \\ ${ }^{1}$ Winogradsky Institute of Microbiology, Research Center of Biotechnology, Russian Academy of \\ Sciences, Moscow, 119071 Russia \\ 2 Topchiev Institute of Petrochemical Synthesis, Russian Academy of Sciences, Moscow, 119991 \\ Russia \\ ORCID
}

Marina Zhurina; 0000-0003-0402-515

Corresponding Author: Marina

Zhurina; email: mzhurik@mail.ru

Dates

Published 13 January 2022

Publishing services provided by Knowledge E

(c) Marina Zhurina et al. This article is distributed under the terms of the Creative Commons Attribution License, which permits unrestricted use and redistribution provided that the original author and source are credited.

Selection and Peer-review under the responsibility of the 8th Scientific and Practical Conference Conference Committee.

\section{G OPEN ACCESS}

Abstract. This study aimed to investigate the dependence of the biocidal activity of polyguanidine (co)polymers on their structure during the formation of biofilms by active PE-degrading cultures of model microorganisms. The Bc-2 copolymer of methacryloyl guanidine hydrochloride (MGHC) and diallyldimethylammonium chloride (DADMAC), which suppressed both the formation of biofilms and the growth of planktonic cultures, exhibited the highest activity. When PE was exposed in tropical soil, the composition of the microbial community on the PE surface differed significantly from that of the community in the surrounding soil. In particular, the proportion of Actinobacteria increased from $7 \%$ to $29 \%$, while the proportion of Bacteroidetes decreased from $38 \%$ to $8 \%$.

Keywords: biofilms, polyhexamethylene guanidine salts, dynamics of biofilm formation, antibiofilm effect, composite materials

\section{Introduction}

Currently, combating microbial biofilms that grow on different artificial surfaces, including plastics, is an urgent task in various sectors of the economics. A wide variety of plastics used for flow systems in medicine and industry are colonized by biofilms. The biofilm biomass alters the flow rate of the system. Moreover, the biofilms formed on catheters and other indwelling medical devices (especially in the case of multi-resistant microbiota), are often associated with infections in patients. As a result, biofilm-resistant materials are being developed worldwide, using different approaches [1].The application 
of biocidal agents is among the methods used to protect polymeric materials from microbial colonization and biodegradation.

The currently used biocidal preparations based on low-molecular-weight substances are cytotoxic. They are dangerous for humans and warm-blooded animals, as well as characterized by a short-term action. Moreover, microorganisms rapidly develop resistance to them. Polymeric biocides, especially guanidine-containing (co)polymers, may be promising [2, 3]. Copolymers composed of various guanidine-containing monomers decrease the probability of the emergence of biocide-resistant microorganisms due to the random inclusion of the monomers. Therefore, the development of new highmolecular non-toxic for humans biocides with prolonged action, the methods for their introduction into polymeric materials, as well as studies of their effects on the microbial growth on polymers, pose significant challenges for the development of approaches to eradicate pathogens and protect materials from biocorrosion. We selectedpolyethylene $(\mathrm{PE})$ as a carrier polymer. PE is a multi-tonnage polymer that has attracted increased attention of researchers due to its poor biodegradability).

To date, polyhexamethylene guanidine (PHMG) salts (Figure 1 shows its general formula) are commercially produced by polycondensation. Currently, PHMG hydrochloride (PHMG-HC) is synthesized by the polycondensation method and characterized by low molecular weight $(\leq 10 \mathrm{kDa})$.

Due to the unique combination of biocidal, physicochemical, and toxicological characteristics, PHMG derivatives are applied in different areas in particular, as a biocidal component of polymer composite materials. [4]. Nowadays, the methods for the synthesis of polyguanidines have been developed. However, little is known about the antibacterial (especially antibiofilm) action of copolymers on microorganisms, which prompted us to study this problem. Our present studies also focus on the formation of biofilms on the surface of composite materials to further test the ability of composite materials that contain biocidal copolymers to inhibit the biofilm growth on their surface.

The method of radical polymerization, which has been developed at Topchiev Institute of Petrochemical Synthesis (Russian Academy of Sciences), makes it possible to synthesize polymers and copolymers of various compositions and structures, with molecular weights of $\geq 1000 \mathrm{kDa}$ [5]. This can significantly affect the properties of polymers, decrease the microbial adaptation to them, and markedly increase the duration of the biocidal action.

This research was devoted to the study of the biocidal properties of polyhexamethylene guanidine hydrochloride (PHMG-HC) and copolymers obtained by radical polymerization. The research is also focused on the development of polyethylene 


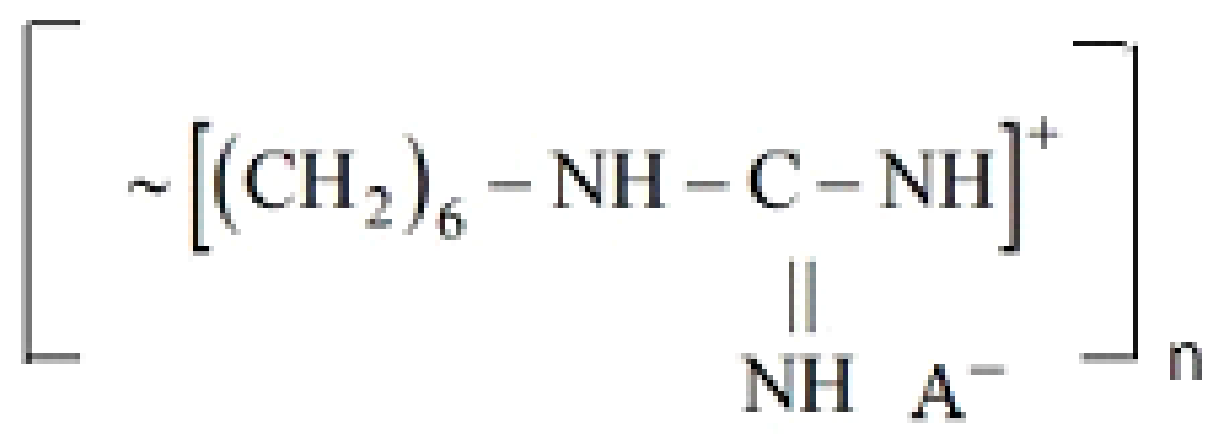

Figure 1: Structural formula of high molecular weight salts of PHMG, where $A$ is the acidic residue of a mineral or organic acid, $\mathrm{n}=30-90$.

nanocomposites containing biocidal components, the study of the effect of the additive composition on the growth of planktonic cultures and microbial biofilms, as well as investigations on biological corrosion of nanocomposites in natural environments.

\section{Materials and Methods}

Preparation of microbial cultures. The following microorganisms obtained from the Collection of Winogradsky Institute of Microbiology (Research Center of Biotechnology, Russian Academy of Sciences) were used in this study: Pseudomonas aeruginosa PAO1, Yarrowia lipolytica 367-3, Kocuria rhizophila 4A-2G and Staphylococcus epidermidis ATCC 14990. The microorganisms were cultured on the LB (Lysogeny Broth) medium (the Lennox formulation; Diaem, Russia). In the experiments with biofilms, 24-h cultures grown in the liquid LB medium on a shaker $(150 \mathrm{rpm})$ at $30^{\circ} \mathrm{C}$ were used as inocula, as described in the earlier papers [6-7].

The study of the effect of polyguanidine copolymers on model cultures was carried out using the modified M9 medium of the following composition ( $\mathrm{g} / \mathrm{L}$ ): $\mathrm{NaCl}, 5 ; \mathrm{KH} 2 \mathrm{PO}$, 3; $\mathrm{NH} 4 \mathrm{Cl}$, 1; glucose, 5; yeast extract, 1; distilled water, $\mathrm{pH}$ 6.5. The medium was autoclaved at $0.5 \mathrm{~atm}$.

To study the effect on the growth of planktonic cultures and biofilms, the following technique was used [8]. Standard chemically pure Teflon cubes (TEKHNOSERVIS, Russia; $3 \mathrm{~g}$ (21 pieces); $4 \times 4 \times 4 \mathrm{~mm}$ in size) served as a carrier for the biofilm growth. The cubes were immersed in the liquid medium $(3 \mathrm{~mL})$, which was supplemented with the inoculum diluted to OD540 $=0.05$. The incubation was carried out for $24 \mathrm{~h}$. At the end of the incubation, it was impossible to directly measure the relative optical density of the planktonic culture due to the colloidal suspension of the polymer in the solution. Therefore, the planktonic culture together with the biofilm was stained with a $0.1 \%$ MTT 
(3-(4,5-dimethylthiazol-2-yl)-2,5-diphenyltetrazolium bromide, Diaem, Russia) solution (3 $\mathrm{mL}$ ) in the LB medium. In the case of the yeast, test tubes were incubated at $33 \otimes \mathrm{C}$ for $60 \mathrm{~min}$; all other cultures were incubat?ed for $20 \mathrm{~min}$. After that, the suspension $(1 \mathrm{~mL})$ was collected and centrifuged to pellet the cells. Formazan was extracted with DMSO $(1 \mathrm{~mL})$ for $24 \mathrm{~h}$, followed by the OD measurements at a wavelength of $590 \mathrm{~nm}$. To stain biofilms, Teflon cubes were rinsed twice with distilled water to remove the residual MTT solution. After that, formazan was extracted with DMSO (3 mL) for $24 \mathrm{~h}$, and OD590 was measured.

The culture growth kinetics in the presence of polyguanidine was studied using an XMark Biorad microplate spectrophotometer. The experiments were carried out in 96-well flat-bottom microplates (TPP, Switzerland). The culture inoculum diluted to OD = $0.05(40 \mu \mathrm{L})$ and the biocide solution were added to each well; the M9 modified medium was supplemented to a final volume of $200 \mu \mathrm{L}$ per well.

Statistics. Experiments were independently repeated at least three times. All numeric data were analyzed using the Mann-Whitney non-parametric test $(p<0.05) /$. As a result of three or more statistical variants for each sample, the median of relative vaues was chosen as an average value. Datawere processed using Microsoft Excel software.

Microscopy. For epifluorescence microscopic analysis, fixed samples of biofilms on PE were stained with SytoTM 9 dye, according to the manufacturer's method (ThermoFisher Scientific, https://www.thermofisher. com/ru/ru/home/life-science/cellanalysis/fluorophores/syto-9.html). Cells were visualized using an Axiolmager.M2 epifluorescence microscope (Carl Zeiss Microscopy $\mathrm{GmbH}$, Germany) equipped with a 503mono digital camera and a Zeiss 38 filter (maximum excitation, $470 \mathrm{~nm}$; maximum emission, $525 \mathrm{~nm}$ ). An immersion objective lens (100x) was used. The images were processed using the ZEN 2 software (Carl Zeiss Microscopy GmbH, Germany). Phase contrast microscopy was performed using an Olympus CX41 microscope (Japan) equipped with an Olympus C5060-ADU camera ( Japan); the ImageScope Lite software package (Image-Scope Color Application, Russia) was used. A sample of PE with biofilms was placed on a glass slide, and a water droplet was added. The preparation, with a coverslip over it, was observed using oil immersion (objective lens, 100x). If required, the photographs were contrasted using the Emboss tool of the Photoshop CS6 Extended software package.

Polyguanidine copolymers were stored as powders. Stock solutions of biocides in distilled water (a concentration of $10 \mathrm{wt} \%$ ) were prepared and stored at $-18 \rrbracket \mathrm{C}$. To standardize the volumes of the liquid added to the microplate wells or the test tubes containing the cubes, serial dilutions of the stock solutions were prepared for the 
TABLE 1: Polyguanidine polymers used in this work.

\begin{tabular}{|c|c|c|c|c|}
\hline $\begin{array}{l}\text { Biocide } \\
\text { number }\end{array}$ & $\begin{array}{l}\text { Copolymer } \\
\text { legend }\end{array}$ & Copolymer name & $\begin{array}{l}\text { Molar ratio } \%, M_{1} \text { : } \\
M_{2}\end{array}$ & $\begin{array}{l}\text { Viscosity, dl/g, } \\
\text { (in } 0.5 \mathrm{~N} \mathrm{NaCl} \text { ) }\end{array}$ \\
\hline $\mathrm{Bc}-1$ & CP-1 & $\begin{array}{l}\text { Copolymer of MGHC }\left(M_{1}\right) \text { and } \\
\text { DADMAC }\left(M_{2}\right)\end{array}$ & $53: 43$ & 0.33 \\
\hline $\mathrm{Bc}-2$ & CP-2 & $\begin{array}{l}\text { Copolymer of MGHC }\left(M_{1}\right) \text { and } \\
\text { DADMAC }\left(M_{2}\right)\end{array}$ & $67: 33$ & 0.95 \\
\hline $\mathrm{Bc}-3$ & CP-3 & $\begin{array}{l}\text { Copolymer of MGHC }\left(M_{1}\right) \text { and } \\
\operatorname{DADMAC}\left(M_{2}\right)\end{array}$ & $77: 23$ & 0.41 \\
\hline $\mathrm{Bc}-4$ & CP-4 & $\begin{array}{l}\text { Copolymer of MGHC }\left(M_{1}\right) \text { and } \\
\text { DADMAC }\left(M_{2}\right)\end{array}$ & $93: 7$ & 0.68 \\
\hline Bc-5 & CP-5 & $\begin{array}{l}\text { Copolymer of MGHC }\left(M_{1}\right) \text { and } \\
\text { DADMAC }\left(M_{2}\right)\end{array}$ & $93: 7$ & 0.34 \\
\hline Bc- 6 & CP-6 & $\begin{array}{l}\text { Copolymer of MGHC }\left(M_{1}\right) \text { and } \\
\text { DADMAC }\left(M_{2}\right)\end{array}$ & $95: 5$ & 0.68 \\
\hline $\mathrm{Bc}-7$ & CP-1.1 & $\begin{array}{l}\text { Copolymer of MGHC }\left(M_{1}\right) \text { and MAAm } \\
\left(M_{2}\right)\end{array}$ & $76: 24$ & 0.89 \\
\hline $\mathrm{Bc}-8$ & CP-1.2 & $\begin{array}{l}\text { Copolymer of MGHC }\left(M_{1}\right) \text { and MAAm } \\
\left(M_{2}\right)\end{array}$ & $38: 62$ & 0.71 \\
\hline Bc-9 & CP-1.3 & $\begin{array}{l}\text { Copolymer of MGHC }\left(M_{1}\right) \text { and MAAm } \\
\left(M_{2}\right)\end{array}$ & $32: 68$ & 1.17 \\
\hline Bc-10 & CP-1.4 & $\begin{array}{l}\text { Copolymer of MGHC }\left(M_{1}\right) \text { and MAAm } \\
\left(M_{2}\right)\end{array}$ & $27: 73$ & 0.37 \\
\hline Bc-11 & CP-2.1 & $\begin{array}{l}\text { Copolymer of MGTFA }\left(M_{1}\right) \text { and } \\
\operatorname{DADMAC}\left(M_{2}\right)\end{array}$ & $90: 10$ & 0.21 \\
\hline Bc-12 & PMAG & Polymethacrylate guanidine & Homopolymer & 0.83 \\
\hline Bc-13 & PMGHC-1 & PolyMGHC & Homopolymer & 0.18 \\
\hline Bc-14 & PMGHC-2 & PolyMGHC & Homopolymer & 1.64 \\
\hline Bc-15 & PMGHC-3 & PolyMGHC & Homopolymer & $>2.0$ \\
\hline Bc-16 & PHMGHC & \multicolumn{3}{|c|}{ Industrially used material } \\
\hline
\end{tabular}

experiments. The composition and some properties of the polyguanidine polymers biocides used in this work are shown in the Table 1.

Preparation and storage of polyethylene samples. . Experimental films were made of PE-277 polyethylene. The films were cut into circles with a diameter of $10 \mathrm{~cm}$ and sterilized by UV treatment for $1 \mathrm{~h}$ (UV lamp DB 30-01 with a power of $30 \mathrm{~W}$, bactericidal power of $6 \mathrm{~W}, 253.7 \mathrm{~nm}$ ). Sterile samples were stored at room temperature.

Exposure in natural conditions. PE samples were delivered sterile to Memyo, Republic of Myanmar (Burma). Some of the samples were placed in the top layer $(5 \mathrm{~cm})$ of Acrisol soil (FAO SOILS PORTAL). Another part of the samples was left above the soil surface so that they were exposed to sunlight. The films were incubated for 180 days, after which they were delivered to the laboratory in sterile containers and examined. 
The mechanical tests polyethylene films were performed on an Instron 1121 tensile testing machine (United Kingdom) at a rate of $5 \mathrm{~mm} / \mathrm{min}$.

Microbiological profiling of communities by the NGS method and bioinformatic data processing were performed by company BioSpark, Russia.

\subsubsection{Results and Discussion}

Experimental medium selection. All studied copolymers were readily soluble in water. However, when added to the culture medium (M9) for testing microorganisms, precipitation and flocs formation occured. To avoid this, the composition of the medium was optimized and the modified M9 medium with minimal precipitation was used for experiments.

The effect of copolymers on the growth of planktonic cultures and biofilms in a liquid medium was investigated. After that, for a group of the most active biocides of a similar structure, culture growth kinetics in a liquid medium were studied and compared with each other. A small amount of a biocide precipitated in the solution interrupted the OD measurement of planktonic cultures; therefore, cells were stained with MTT along with biofilms and the metabolic activity was measured.

\subsection{Effect the biocides on planktonic cells and biofilms on model cultures}

Three microorganisms were used - the Gram-negative bacterium P. aeruginosa PAO1, the yeast Y. lipolytica 367-3, and the Gram-positive bacterium S. epidermidis ATCC 14990. To compare the inhibitory effect of polyguanidine compounds on biofilm formation and planktonic growth, tenfold dilutions of biocides were used. As a result of these preliminary studies, a concentration of $0.1 \%$ by weight was chosen. In $1 \%$ concentration all biocides significantly suppressed the growth of microorganisms, and in $0.01 \%$ concentration, the suppression was insignificant, so it was impossible to compare the effectiveness of biocides with each other. The results are presented in Table 2 . The most promising were $\mathrm{Bc}-1$ - Bc-6, copolymers of MGHC and DADMAC (Bc-16 is an industrially used polymer with high activity and taken as a control.) It was shown that these biocides do not completely inhibit the growth of planktonic cultures, but they significantly inhibit the growth of biofilms. We assumed that polyguanidines in solution are adsorbed on teflon surface and directly prevent biofilm formation, but experimental verification did not support this hypothesis. 
TABLE 2: The effect of $0.1 \%$ biocide solutions on the growth of biofilms and planktonic cultures.

\begin{tabular}{|c|c|c|c|c|c|c|}
\hline & $\begin{array}{l}\text { S.epidermidis } \\
\text { ATCC } 14990\end{array}$ & & $\begin{array}{l}\text { Y.lipolytica } \\
367-3\end{array}$ & & $\begin{array}{l}\text { P.aeruginosa } \\
\text { PAO1 }\end{array}$ & \\
\hline № of a biocide & $\begin{array}{l}\text { Planktonic } \\
\text { cells }\end{array}$ & biofilms & $\begin{array}{l}\text { Planktonic } \\
\text { cells }\end{array}$ & biofilms & $\begin{array}{l}\text { Planktonic } \\
\text { cells }\end{array}$ & biofilms \\
\hline $\mathrm{Bc}-1$ & 24 & 0 & 56 & 60 & 0 & 0 \\
\hline Bc -2 & 24 & 0 & 64 & 45 & 0 & 0 \\
\hline $\mathrm{BC}-3$ & 27 & 0 & 61 & 34 & 0 & 0 \\
\hline Bc-4 & 24 & 0 & 67 & 33 & 0 & 0 \\
\hline Bc-5 & 28 & 0 & 65 & 35 & 0 & 0 \\
\hline $\mathrm{Bc}-6$ & 48 & 22 & 78 & 153 & 43 & 18 \\
\hline $\mathrm{Bc}-7$ & 33 & 0 & 87 & 85 & 50 & 35 \\
\hline $\mathrm{Bc}-8$ & 0 & 70 & 0 & 236 & 0 & 46 \\
\hline $\mathrm{Bc}-9$ & 0 & 0 & 0 & 25 & 0 & 28 \\
\hline Bc-10 & 0 & 78 & 0 & 114 & 0 & 61 \\
\hline Bc-11 & 30 & 0 & 65 & 40 & 0 & 0 \\
\hline Bc-12 & 12 & 97 & 0 & 63 & 102 & 90 \\
\hline Bc-13 & 31 & 0 & 95 & 144 & 0 & 0 \\
\hline Bc-14 & 0 & 29 & 0 & 566 & 7 & 73 \\
\hline Bc-15 & 57 & 6 & 126 & 95 & 76 & 63 \\
\hline Bc-16 & 0 & 0 & 0 & 0 & 0 & 0 \\
\hline
\end{tabular}

Biocides inhibition of bacterial growth, \% of control. In control samples (100\%) the metabolic activity of planktonic cells or biofilms was measured in absence of biocides.

To compare the efficiency of these six different MGHC and DADMAC copolymers, the culture growth was tested in dynamics in the presence of the biocide. The experiments were carried out with the same three model cultures: the Gram-negative bacterium $P$. aeruginosa PAO1, the yeast Y. lipolytica 367-3, and the Gram-positive bacterium S. epidermidis ATCC 14990. Lower concentrations of biocides (0.01 and $0.001 \%$ ) were used. Cultures without biocide additives were used as control. The microplate spectrophotometer does not allow for the separate determination of biofilms and planktonic cells of a culture. Thus, these results reflect the summarized plankton and biofilm components in the dynamics of culture growth.

\subsection{Culture Growth Kinetics in the Presence of Biocides}

Effect of biocides on the growth of S. epidermidis ATCC 14990. Out of six biocides tested, Bc-2 exhibited the most pronounced inhibitory effect on the growth of S. epidermidis ATCC 14990. It completely inhibited the growth of cultures at a concentration of $0.01 \%$ in the medium (Figure 2 , red curve). At the same time for other studied Bc2 concentrations, this biocide had a similar effect independently of its content in the 


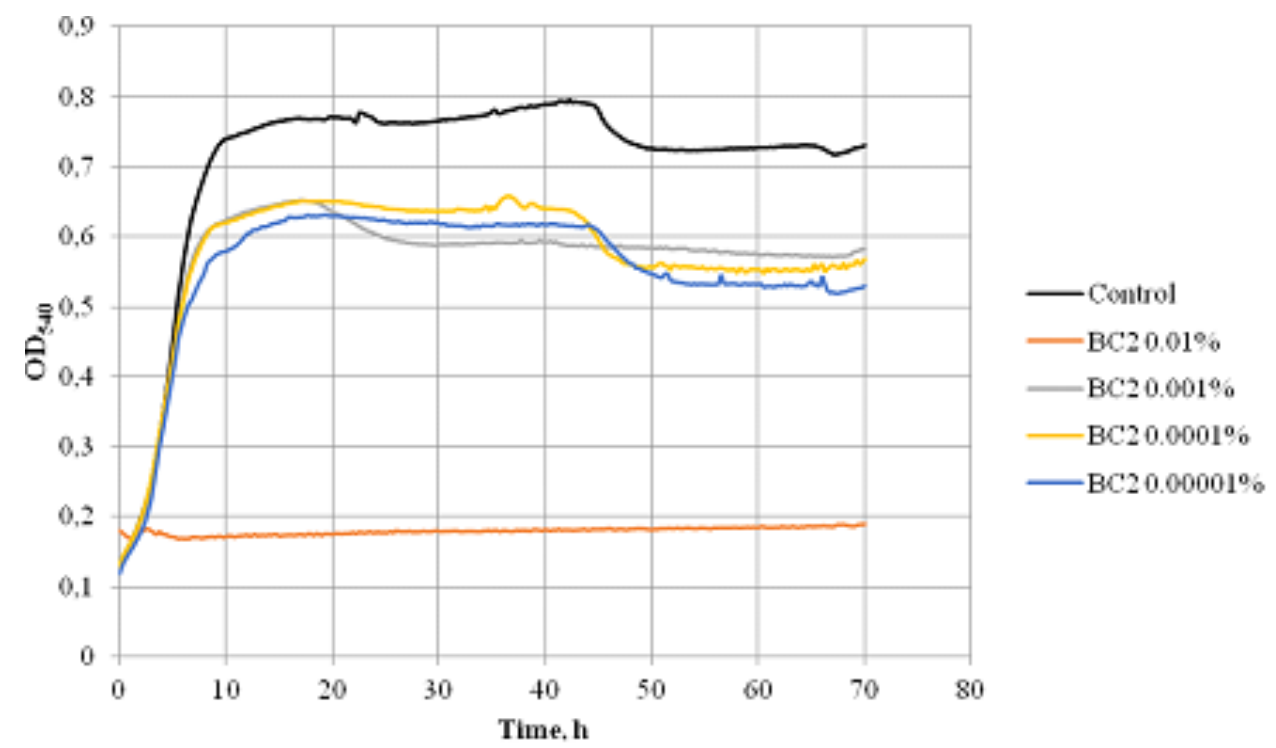

Figure 2: Effect of Bc-2 on the growth of S. epidermidis ATCC 14990.

medium (Figure 2, grey, yelloy and blue curves). For instance, the maximum $\mathrm{OD}_{540}$ of the cultures varied within $79-82 \%$ of the control, while the shape and slope of the curves did not change. At the highest concentrations, biocides 1, 3, and 4 had a significant inhibitory effect on the growth of the epidermal staphylococcus $(65,43$, and 39.5\% of the control, respectively). Nevertheless, a complete suppression of growth was not observed. Moreover, Bc-1 and Bc-3 significantly extended the lag phase (32 and $40 \mathrm{~h}$, respectively) that preceded an active growth of $S$. epidermidis. Other biocides did not prolong the lag phase or extended it insignificantly (for instance, in the case of BC- 6 $(0.01 \%)$, the duration of the lag phase was $4.5 \mathrm{~h})$. No lag phase was recorded for the control samples. Biocides 5 and 6 had the least inhibitory effect on S. epidermidis ATCC 14990 (the maximum OD was $82 \%$ of $t$ he control at the highest concentrations of both biocides).

Effect of biocides on the growth of Y. lipolytica 367-3. In general, the effects of the biocides on the yeast $Y$. lipolytica 367-3 were similar to those on S. epidermidis ATCC 14990. The most pronounced effect was observed at the highest concentrations of biocides. The strongest inhibitory effect was noted for Bc-2 (Figure 3) when the highest $O_{540}$ was $19.5 \%$ of the control. At the same time, the lag phase preceding the growth was approximately $32 \mathrm{~h}$. Out of all biocides tested, Bc- 6 had the lowest inhibitory effect. At a concentration of $0.01 \%$, the highest OD in the samples reached $70 \%$ of the control OD. Although the maximum OD was lower in the presence of BC- 6 than BC-5 (74\%), Bc-6 did not extend the lag phase significantly (in contrast to BC-5). The duration of the lag phase was 12.5 and $5.5 \mathrm{~h}$ in the presence of Bc-5 and Bc-6, respectively. Bc-1 also exhibited a strong inhibitory effect: the highest OD was only $26.5 \%$ of the 


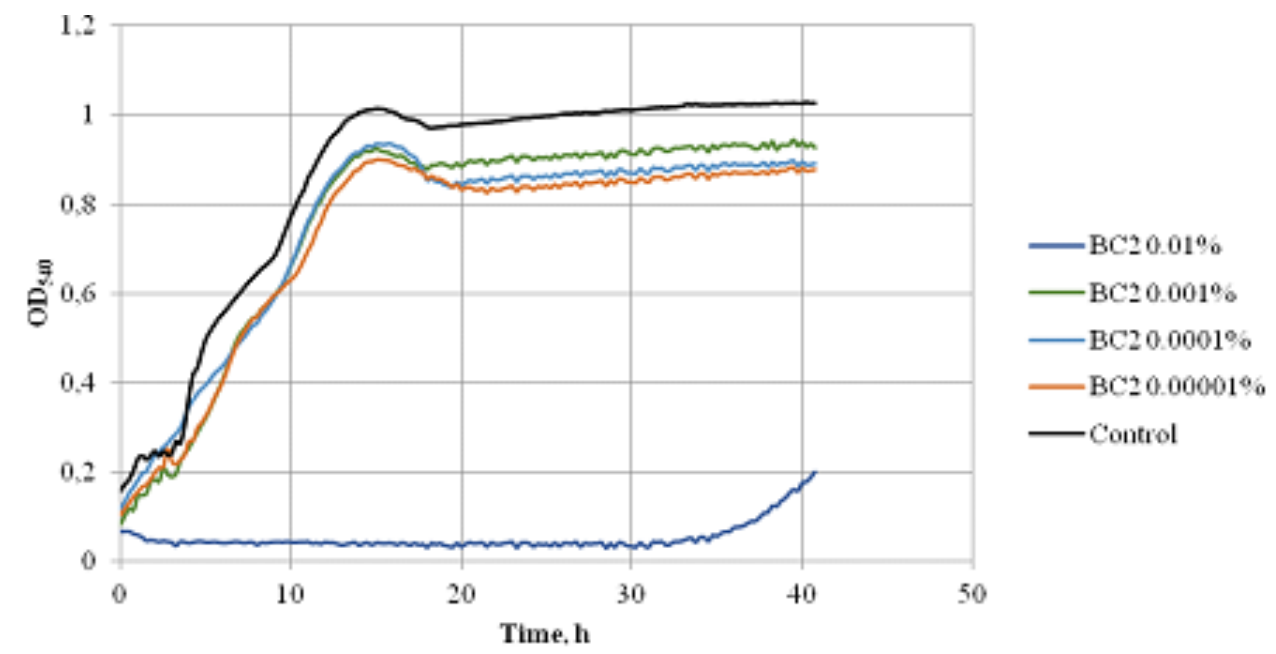

Figure 3: Effect of Bc-2 on the growth of Y. lipolytica 367-3.

control. Moreover, the growth curve was equally inclined (relative to the abscissa axis) throughout the entire cultivation period, i.e., no remarkable lag phase was observed.

Effect of biocides on the growth of P. aeruginosa PAO1. In contrast to the previously mentioned microorganisms (the Gram-positive bacterium S. epidermidis ATCC 14990 and the yeast $Y$. lipolytica 367-3), the growth of the Gram-negative bacterium P. aeruginosa PAO1 was less inhibited. At the highest concentrations, the biocides either slightly suppressed the growth (for instance, BC-1) or did not affect it (Bc-6). Bc-1 and Bc-2, which were the most efficient biocides (at a concentration of $0.01 \%$ in the medium) against other microorganisms studied, inhibited the growth of the Pseudomonas culture at least at the beginning of cultivation. However, at the end of incubation, the maximum OD was either at the level of that of the control sample or slightly higher than the control OD. Low concentrations of testing biocides did not suppress the Pseudomonas growth.

Thus, according to the results of our study, Bc-2 is the most promising anti-biofilm agent. To study the effect of the biocide in the polymer matrix, it is important to understand the process of the biofilm formation on a polymer carrier in the absence of the biocide. Microorganisms form microcolonies on the polyethylene surface. This process has been described in detail in our recent study [9]. For instance, Figure. 4 illustrates a three-species biofilm on the PE surface.

\subsection{Polymeric materials in natural conditions}

To understand the mechanisms of biocorrosion, , it is necessary to study not only biofilms of model microorganisms, but also to identify the PE-colonizing microorganisms and 

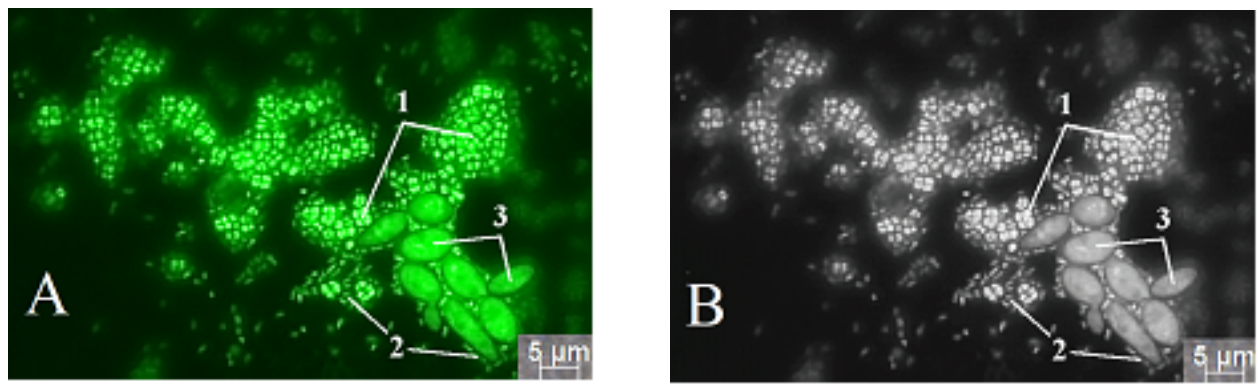

Figure 4: Triple-species biofilms on PE film surface. Phase contrast (A) and embossing (B) (this emage were contrasted using the Emboss tool of the Photoshop CS6 Extended software package. Levenhuk DTX 500 digital microscope [Adapted from 9]. 1 - Y. lipolytica 367-3, P. aeruginosa PA01, 3- K. rhizophila 4A-2G.

estimate the plastic destruction in natural conditions. Thus, we carried out a 180-day exposure of PE samples in soil.

The exposition of polymeric materials in soil was performed in Myanmar (Burma), Memyoyu. This region was chosen because tropical soils contain a variety of destructive microorganisms [10], and the exposure conditions (elevated temperature and humidity in a subequatorial climate) are favorable for the formation of biofilms on the surface of polyethylene.

After the exposure, the PE films were examined visually, and their physico-mechanical properties were investigated. In addition, we analyzed the community of microorganisms that formed a biofilm on the surface of PE from the soil and compared this community with the community of microorganisms from a soil sample in same area but had no contact with polyethylene.

Visual examination of the surface of the samples after 180 days of exposure showed that during aging in air, PE-277 degraded more intensively than in soil. This is due to solar irradiation from which the samples in the soil were sheltered. Upon exposure in soil, the samples became darker colored.

The samples of PE-277 were determined to change physical and mechanical properties: the greatest decrease in physical and mechanical durability occured when exposed to air. In the soil, the properties of PE-277 practically did not change with aging.

\subsection{6-S RNA methagenomic profile of microbial communities (Figure 5)}

Totally 67961 sequences were isolated from soil and 8829 from the PE surface. 4180 and 2098 OTUs numbers were formed from them respectively. In both analyzed libraries of 16S rRNA gene fragments obtained from high-throughput sequencing, not only bacterial, 
but also archaeal components were detected in a small amount, $0.2 \%$ and $0.05 \%$ of the total microbial number on PE and soil samples, respectively.

Phylogenetic diversity of bacterial communities isolated from a soil sample. According to taxonomic phylotype distribution of bacteria at the phyla level, the microbial community of soil samples consists of Bacteroidetes (38\% of all bacteria), including Sphingobacteria (55\% of bacteroids) Cytophagia 34\% and Flavobacteria 7\%; Proteobacteria (36\% of all bacteria in the soil), including Alphaproteobacteria (46\%), Betaproteobacteria (16\%), Gammaproteobacteria (18\%, Deltaproteobacteria (16\%); and in addition, Actinobacteria 7\%, Verrucomicrobia 5\%, Chloroflexi 5\%, Acidobacteria 4\%, Cyanobacteria $1 \%$, Firmicutes $1 \%$, Saccharibacteria $1 \%$ and the rest $2 \%$.

Phylogenetic diversity of bacterial communities isolated from surface of 180-day exposed PE. Study of taxonomic phylotype distribution of bacteria at the phyla level in PE surface shown the following composition of the community: Bacteroidetes (8\% of all bacteria), including Sphingobacteriia (55\% of bacteroids) Cytophagia $29 \%$ and Flavobacteriia 8\%; Proteobacteria (36\% of all bacteria in the soil), including Alphaproteobacteria (50\%), Betaproteobacteria (15\%), Gammaproteobacteria (15\%), Deltaproteobacteria (20\%); Acidobacteria 9\%, Actinobacteria 29\%, Saccharibacteria 6\%, Chloroflexi 5\%, Firmicutes 3\%, Verrucomicrobia 2\%, Gemmatimonadetes $1 \%$ and the rest $1 \%$.

The results show that, in comparison with the soil sample, the community of microorganisms on PE surface contains a higher ratio of actinobacteria (percentage increases from 7 in soil to $29 \%$ on PE). Percentage of Bacteroidetes decreases from $38 \%$ to $8 \%$. The ratio of the remaining microorganisms did not change significantly.

\section{Conclusion}

Thus, according to the results of our study, Bc-2 is the most promising antibiofilm agent for production composite material from polyethylene.

A - Community from PE surface after exposition in the soil; B - Community from the soil witout PE.

\section{Acknowledgment}

This work was supported by the Russian Foundation for Basic Research, project no. mk18-29-05048 and partially supported by the Ministry of Science and Higher Education of the Russian Federation. 

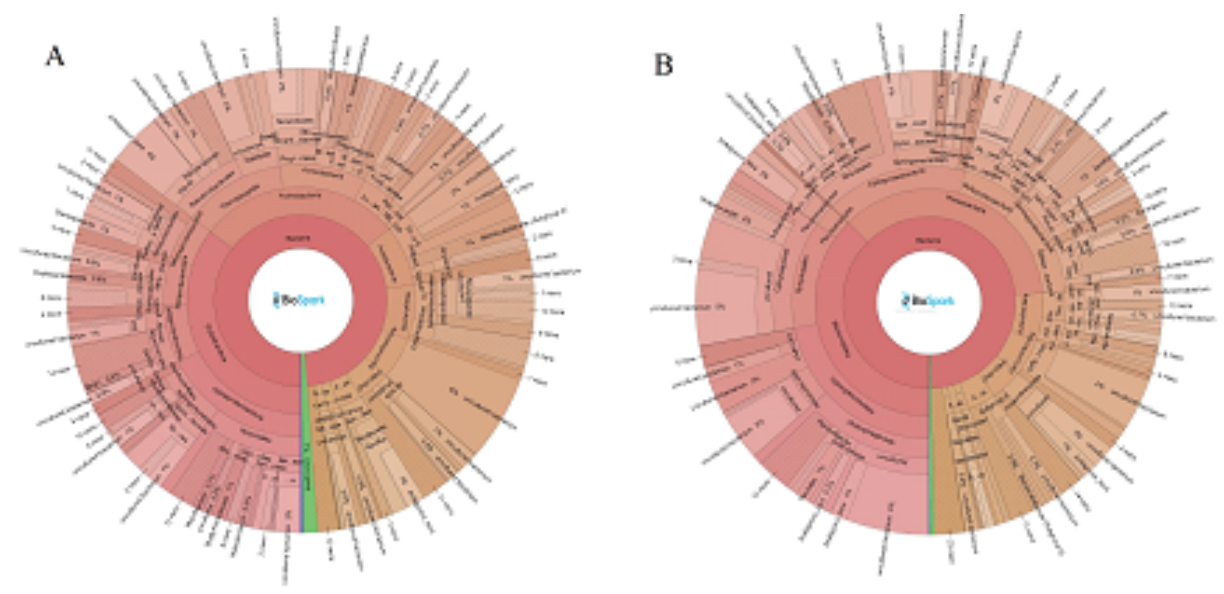

Figure 5: Diagram illustrating species diversity of microbial community.

\section{Compliance with Ethical Standards}

The authors declare no conflict of interest. This article does not contain any studies involving animals or human participants performed by the authors.

\section{References}

[1] Ramasamy M, Lee J. (2016). Recent nanotechnology approaches for prevention and treatment of biofilm-associated infections on medical devices. BioMed Research International. 2016;2016:1851242-1851242. DOI: 10.1155/2016/1851242

[2] Brzezinska SM, Walczak M, Jankiewizs $U$ et al. Antimicrobial activity of polyhexamethylene guanidine derivatives introduced into polycaprolactone. Journal of Polymers and the Environment. 2018;26:589-595.

[3] Ghamrawi S, Bouchara J-P, Tarasyuk O et al. Promising silicones modified with cationic biocides for the development of antimicrobial medical devices. Materials Science and Engineering. 2017; 75:969-979.

[4] Gerasin VA, Mendeleev DI, Kurenkov VV et al. Guanidine-containing organomineral complexes as biocide additives to polymeric composites. Russian Journal of Applied Chemistry. 2018;91:1297-1304.

[5] Sivov NA. Biocide guanidine containing polymers: Synthesis, structure and properties. Moscow, Russia, CRC Press; 2006.

[6] Mart'yanov SV, Zhurina MV, El' Registan Gl et al. Activation and prevention of formation of bacterial biofilms by azithromycin. Microbiology (Moscow). 2014;83(6):723-731. 
[7] Gannesen AV, Zhurina MV, Veselova MA, et al. Regulation of biofilm formation by Pseudomonas chlororaphis in an in vitro system. Microbiology. 2015;84:319-327.

[8] Plakunov VK, Mart'yanov SV, Teteneva NA et al. A universal method for quantitative characterization of growth and metabolic activity of microbial biofilms in static models. Microbiology. 2016;85:509-513.

[9] Zhurina MV, Kallistova AYu, Panyushkina AE et al. Specific features of formation of multispecies microbial biofilms on polyethylene surface. Microbiology. 2020;89(40):396-404.

[10] Divyalakshmi S, Subhashini A. Screening and isolation of polyethylene degrading bacteria from various soil environments. IOSR Journal of Environmental Science, Toxicology and Food Technology. 2016;10:1-7. 